\title{
Color Cartographic Pattern Recognition Using the Coarse to Fine Scale Method
}

\author{
Efrén González-Gómez and Serguei Levachkine \\ Centre for Computing Research (CIC) - National Polytechnic Institute (IPN) \\ UPALMZ, CIC Building, 07738, Mexico City, Mexico \\ efren@esfm.ipn.mx, sergei@cic.ipn.mx
}

\begin{abstract}
Hard problem of cartographic pattern recognition in fine scale ${ }^{1}$ maps, using information that comes from coarse scale ${ }^{2}$ maps, is considered. The maps are raster-scanned color maps of different thematic, representing the same territory in coarse and fine scale respectively. A solution called Coarse-to-Fine Scale Method is proposed. This method is defined in terms of means: coarse scale maps and their information; concepts: image associated function, cartographic knowledge domain and cartographic pattern; and tools: a set of clustering criteria of the Logical Combinatorial Pattern Recognition.
\end{abstract}

\section{Introduction. Formal Statement of the Problem}

In this paper we present the main ideas of the C2FS (Coarse-to-Fine Scale) method of color cartographic pattern recognition. This method has been originated from unsolved problem of the vector description of raster objects. To date, it is hard to see the ways to obtain even a partial, but general, i.e. applied to any type of raster objects, solution of the problem; see survey papers [2][4][7] for detail discussion. In certain sense, the C2FS method represents a promising alternative.

Let us suppose that we have a vector image $\mathrm{I}_{1}$ (or already recognized raster image) in scale $1: s_{1}$ of a given territory $\mathbf{T}$ and a raster image $I_{2}$ of $\mathbf{T}$ to be vectored (recognized) in scale $1: s_{2}$, and $s_{1}>s_{2}$ (e.g. $s_{1}=100,000$ and $s_{2}=50,000$ ). Our goal is to use the information from $I_{1}$ in vectorization of $I_{2}$. Note that $I_{1}$ can be considered as a "generalization" 3 of $I_{2}: I_{1}=G\left(I_{2}\right)$, i.e. if an object $O_{2} \in I_{2}$, then there can exist $O_{1} \in$ $\mathrm{I}_{1}$, such that $\mathrm{O}_{1}=\mathrm{G}\left(\mathrm{O}_{2}\right)$. We denote $\Omega$ - the set of all such objects $\mathrm{O}_{2}$ from $\mathrm{I}_{2}$ and $\Theta$ the compliment of $\Omega$ in $\mathrm{I}_{2}: \mathrm{I}_{2}=\Omega \cup \Theta$. We also put $\omega=\mathrm{G}(\Omega)$ and note that $\omega \subseteq \mathrm{I}_{1}$. Obviously, to vector objects from $\Omega$ and $\Theta$ we need two different strategies. The objects from $\Omega$ can be vectored, using the features (position, color or colors, shape, etc.) of the vector objects from $\omega$. After, the objects of $\Omega$ have being vectored we can vector the objects from $\Theta$ by one of the recognition modules [2][7] as a "new" cartographic material.

\footnotetext{
1 Inferior to $1: 200,000$.

2 Superior to $1: 200,000$.

3 We do not discuss here what this generalization is.
} 


\section{Theoretical Background}

The theoretical background of the C2FS method is based on the following definitions:

Definition 1. A digital image is a matrix $\|M(i, j)\|_{n \times m}$ such that $0 \leq \mathrm{i} \leq \mathrm{n}, 0 \leq \mathrm{j} \leq \mathrm{m}$; and $\mathrm{M}(\mathrm{i}, \mathrm{j})=(\mathrm{r}, \mathrm{g}, \mathrm{b})$, where $\mathrm{r}, \mathrm{g}, \mathrm{b}$ are elements of the set $\{0,1, \ldots, 255\}$.

Definition 2. Let $\mathbf{I}$ be a digital image according to the definition 1, then Image Associated Function (IAF) $f_{\mathrm{I}}: \mathrm{Z} \times \mathrm{Z} ' \rightarrow \mathrm{M}$ to $\mathbf{I}$ is defined as follows:

a) IAF domain is $Z \times Z^{\prime}$ where $Z=\{0,1, \ldots, n\}$ and $Z^{\prime}=\{0,1, \ldots, m\}$.

b) IAF co-domain is a set $\mathrm{M}$ without repeated elements, which is composed of the elements of $\mathbf{M}(\mathrm{i}, \mathrm{j}) \in \mathbf{I}$.

c) IAF is assigned to each pair $(i, j)$ in $Z \times Z^{\prime}$ the corresponding value given by the matrix $\|\mathrm{M}(\mathrm{i}, \mathrm{j})\|$. In other words, $f_{\mathrm{I}}(\mathrm{i}, \mathrm{j})=\mathrm{M}(\mathrm{i}, \mathrm{j}), \forall(\mathrm{i}, \mathrm{j}) \in \mathrm{Z} \times \mathrm{Z}^{\prime}$.

Definition 3. Cartographic Knowledge Domain (CKD) is a finite space of attributive, topologic, logical and spatial data, which are associated with raster cartographic objects presented in maps [9] ${ }^{4}$.

Definition 4. Let $\mathbf{I}$ be a digital image according to definition 1 and let $f_{\mathrm{I}}: Z^{\prime} Z^{\prime} \rightarrow M$ be IAF. Cartographic Pattern (CP) $\mathbf{P}$ in $\mathbf{I}$ is a function such that

1) $\mathbf{P}=\left.f_{\mathrm{I}}\right|_{\mathrm{Zp}}: \mathrm{Z}_{\mathrm{p}} \rightarrow \mathrm{M}_{\mathrm{p}}$, where $\mathrm{Z}_{\mathrm{p}} \subseteq \mathrm{Z} \times \mathrm{Z}$ ' and $\mathrm{M}_{\mathrm{p}} \subseteq \mathrm{M}$. In other words, $\mathbf{P}$ is the restriction of IAF $f_{\mathrm{I}}$ to some set $Z_{\mathrm{p}}$.

2) A concept from $C K D$ can be assigned to the set $Z_{p}$ or $M_{p}$.

Whereas $\mathbf{P}$ only fulfills condition 1 , it is a candidate to be a CP.

These definitions are served as a compliment to clustering criteria coming from Logical Combinatorial Pattern Recognition: $\beta_{0}$-Connected, $\beta_{0}$-Compacted, $\beta_{0}$ Complete Maximal [1][3] to segment and recognize the image. We apply the clustering criteria only to IAF co-domain because this contains much less elements than the total number of pixels presented in the digital image (raster map).

Clustering criteria. Next, we analyze mentioned above clustering criteria and the similarity measures in application to color cartographic image processing. To our knowledge, this is one of the first works in this direction; see [2][3][8] for belief.

We use the following notations. Let $\mathbf{I}$ be a digital cartographic image; $f_{\mathrm{I}}$ be an Image Associated Function, $f_{\mathrm{I}}: \mathrm{Z} \times \mathrm{Z}^{\prime} \rightarrow \mathrm{M} ; \Gamma: \mathrm{U} \times \mathrm{U} \rightarrow[0,1]$ be a Similarity Function, where $U$ is a finite space of objects to classify; $\beta_{0}$ be nonnegative real number.

A subset $G_{i}$ in $U$ is called $\beta_{0}$-Connected, if $\forall O_{r}, O_{s} \in G_{i}$, then there exist $\mathrm{O}_{\mathrm{i}_{1}}, \ldots, \mathrm{O}_{\mathrm{i}_{\mathrm{q}}} \in \mathrm{G}_{\mathrm{i}}$ such that $\mathrm{O}_{\mathrm{r}}=\mathrm{O}_{\mathrm{i}_{1}}, \mathrm{O}_{\mathrm{s}}=\mathrm{O}_{\mathrm{i}_{\mathrm{q}}}$ and $\forall \mathrm{p} \in\{1, \ldots, \mathrm{q}-1\} \Gamma\left(\mathrm{O}_{\mathrm{i}_{\mathrm{p}}}, \mathrm{O}_{\mathrm{i}_{\mathrm{p}+1}}\right) \geq \beta_{0}$. Moreover, if $\mathrm{O}_{\mathrm{k}} \in \mathrm{U}$ and $\exists \mathrm{O}_{\mathrm{j}} \in \mathrm{G}_{\mathrm{i}}$ such that $\Gamma\left(\mathrm{O}_{\mathrm{k}}, \mathrm{O}_{\mathrm{j}}\right) \geq \beta_{0}$ then $\mathrm{O}_{\mathrm{k}} \in \mathrm{G}_{\mathrm{i}}$.

In cartographic digital images, this criterion can be used to find groups in codomain $\mathrm{M}$, defined by the elements that allow a gradual transition in terms of the

${ }^{4}$ For example, attributive data like type of border, name of state, surface, population, administrative unit, among others; geometric data like contours, coordinates, etc. [2]. 
similarity function. While for cartographic patterns, this can be used when the patterns are defined by colors that go from "clear" blue to "dark" blue, for instance. A problem comes when there are gradual transitions between colors in the image and each color of that transition is used to represent a different cartographic pattern. Notice that this problem depends on the image context and can be solved by using other two criteria that are defined in the following.

A subset $G_{i}$ in $U$ is called $\beta_{0}$-Compacted, if $\forall \mathrm{O}_{j} \in G_{i} \exists O_{i} \in G_{i}$ such that $O_{i} \neq O_{j}$ and $\Gamma\left(\mathrm{O}_{\mathrm{i}}, \mathrm{O}_{\mathrm{j}}\right)$ takes the maximum value.

In other words, the objects in a $\beta_{0}$-Compacted set are most similar objects with respect to the similarity function. As we have already mentioned, the applications of $\beta_{0^{-}}$ Connected criterion depend on the image context. To make it context-independent, we can successively apply the $\beta_{0}$-Compacted criterion and/or the following criterion.

A subset $\mathrm{G}_{\mathrm{i}}$ in $\mathrm{U}$ is called $\beta_{0}$-Complete Maximal iff $\forall \mathrm{O}_{\mathrm{i}}, \mathrm{O}_{\mathrm{j}} \in \mathrm{G}_{\mathrm{i}}$, the similarity function $\Gamma$ computed for both elements is greater or equal than $\beta_{0}$, and if $\Gamma\left(\mathrm{O}_{\mathrm{k}}, \mathrm{O}_{\mathrm{p}}\right) \geq$ $\beta_{0}$, where $\mathrm{O}_{\mathrm{k}} \in \mathrm{G}_{\mathrm{i}}$, then $\mathrm{O}_{\mathrm{p}} \in \mathrm{G}_{\mathrm{i}}$.

According to our experiments, this criterion has a disadvantage that if it is applied to the digital image before other two criteria it can generate more than necessary groups in the co-domain. On the other hand, it can be applied to $\beta_{0}$-Connected groups iteratively extracting more precisely the groups of interest (finer clustering); that is its advantage. Once again, the use of each single criterion depends on the image context, while their combination may not.

Summing-up the clustering criteria analysis, we note the following:

- Elements of the IAF co-domain can be grouped/structured in different groups depending on the clustering criteria.

- Groups/structures obtained by the application of a criterion $\Pi^{5}$ can result in the union of groups/structures formed by another criterion $\Pi$ '. In other words, successive application of clustering criteria leads to gradually finer structuring of the IAF co-domain. Thus, a hierarchy under inclusion relation between the groups is settled down, in which "general" ("coarse") groups are at superior level $\left(\beta_{\mathrm{o}}\right.$ Connected criterion) and "specific" ("fine") groups are at inferior level ( $\beta_{\mathrm{o}}-\mathrm{Com}$ pacted and/or $\beta_{\mathrm{o}}$ - Complete Maximal criteria) [1].

On the other hand, these criteria are general and abstract. To apply them in image processing, we should make them specific and concrete for the pixel clustering. Thus, we use a criterion of similarity and modified HSI color space. Indeed, in normal HSI space, if the intensity is equal to zero then the saturation is indeterminate, and if the saturation is zero then hue is indeterminate. In the modified HSI space, we remove the singularities, i.e. intervals where the saturation and hue are indeterminate ${ }^{6}$.

$5 \Pi, \Pi$ ' is one of the three criteria: $\beta_{0}$-Connected, $\beta_{0}$-Compacted or $\beta_{0}$-Complete Maximal.

6 More general approach to modified non-linear color spaces see in [6]. 
Similarity criterion and similarity function. Let $\mathbf{I}$ be a cartographic digital image. Let $\mathrm{f}_{\mathbf{I}}: \mathrm{Z} \times \mathrm{Z} ' \rightarrow \mathrm{M}$ be IAF. The Similarity Criterion $\mathrm{C}: \mathrm{M} \times \mathrm{M} \rightarrow[0,1]$ is defined as follows: let $\mathrm{p}, \mathrm{q} \in \mathrm{M}$; we denote by $\mathrm{I}(\mathrm{k}), \mathrm{S}(\mathrm{k})$ and $\mathrm{H}(\mathrm{k})$ the intensity, saturation and hue respectively of some point $\mathrm{k} \in \mathrm{M}$, then $\mathrm{C}(\mathrm{p}, \mathrm{q})$ :

$C(p, q)=\left\{\begin{array}{c}C_{1}(p, q)=\left\{\begin{array}{c}C_{3}(q) \text { if } I(p) \leq N \\ C_{4}(p, q) \text { otherwise }\end{array} \quad \text { if }|I(p)-I(q)| \leq C\right. \\ 0\end{array}\right.$

where :

$C_{3}(q)=\left\{\begin{array}{l}1 \text { if } I(q) \leq N \\ 0 \text { otherwise }\end{array}\right.$

$C_{4}(p, q)=\left\{\begin{array}{l}C_{5}(q) \text { if } I(p) \geq B \\ C_{6}(p, q) \text { otherwise }\end{array}\right.$

$C_{5}(q)=\left\{\begin{array}{l}1 \text { if } I(q) \geq B \\ 0 \text { otherwise }\end{array}\right.$

$C_{6}(p, q)=\left\{\begin{array}{l}C_{7}(q) \text { if } S(p) \leq R \quad y \quad I(q) \neq 0 \\ 1-|I(p)-I(q)| \text { If } S(p) \leq 0.03 \text { and } I(q)=0 \quad \text { or } \quad S(q) \leq 0.03 \\ 1-\sqrt{(S(p) \cos (H(p))-S(q) \cos (H(q)))^{2}+(S(p) \sin (H(p))-S(q) \sin (H(q)))^{2}} \text { otherwise }\end{array}\right.$

$C_{7}(q)=\left\{\begin{array}{l}1 \text { if } S(q) \leq R \\ 0 \text { otherwise }\end{array}\right.$

Using this similarity criterion, it is possible to define the Similarity Function only in terms of this criterion. Unique feature used in definition of $C$ is color. However, we can define another criterion $C_{a}$ and consider other features besides of color, constructing $C_{a}$ and corresponding similarity function.

According to the similarity criterion above, the similarity function coincides with the criterion $\mathrm{C}(\mathrm{p}, \mathrm{q}) . \mathrm{C}(\mathrm{p}, \mathrm{q})$ for elements $\mathrm{p}$, $\mathrm{q}$ from the IAF co-domain are computed as follows; refer formulas above.

a) If the absolute value of difference between their intensities is more than some threshold $\mathrm{C}$ the value of similarity function is equal to zero.

b) Otherwise, if this value is less than $\mathrm{C}$, then if both intensities are less or equal than some another threshold N (average of all colors close to "black") or the difference is more or equal than yet another threshold B (average of all colors close to "white"), then the value of similarity function is equal to one ${ }^{7}$

c) If $\neg \mathrm{a} \wedge \neg \mathrm{b}$, then the saturation of $\mathrm{p}$ and $\mathrm{q}$ are considered as follows.

7 This can be interpreted as averaging of clear and dark tonalities. 
c1) If both saturation are less or equal than some threshold $\mathrm{R}$, then the value of similarity function is equal to one.

c2) If one of saturation is less or equal than $R$, then the value of similarity function is equal to one minus the absolute value of the difference of intensities.

c3) If $\neg \mathrm{c} 1 \wedge \neg \mathrm{c} 2$, then the saturation as well as hue are determinate, forming two vectors for $\mathrm{p}$ and $\mathrm{q}$ in polar coordinates (saturation (radii), hue (angle)). In this case, the value of similarity function is equal to one minus the Euclidean distance between these two vectors; see also formula above.

Figure 1 shows the results obtained with the application of the clustering criteria (first $\beta_{0}$-Connected and then $\beta_{0}$-Complete Maximal), which use just defined the similarity function, to segment a complex color image.

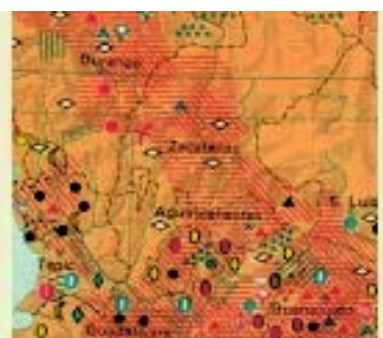

(a)

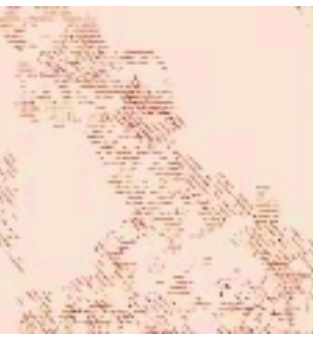

(b)

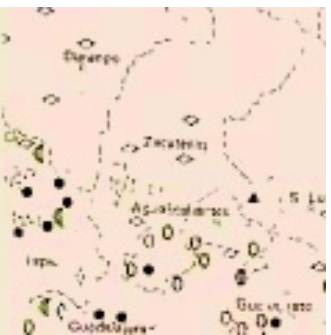

(c)

Fig. 1. (a) Source Image. (b) and (c) Segmentation of the objects of interest.

\section{Application of the C2FS Method to Cartographic Pattern Recognition}

Let $\mathbf{I}$ be a digital cartographic image $\|\mathrm{M}(\mathrm{i}, \mathrm{j})\|_{\mathrm{n} \times \mathrm{m}}$. First we should compute the Image Associated Function as follows. Let $Z=\{0, \ldots, n\}, Z^{\prime}=\{0, \ldots, m\}$, consider the Cartesian product $Z \times Z^{\prime}$ and the difference of the sets $A$ and $B: A-B=\{x \in A \mid x \notin$ B \}. Next the following steps are employed:

1. Let $\mathrm{p}_{\mathrm{o}}=0, \mathrm{q}_{\mathrm{o}}=0$, then the sets are defined:

$$
\begin{aligned}
& G_{0}=\left\{M(x, y) \mid M(x, y)=M\left(p_{o}, q_{o}\right)\right\} \text { and } \\
& G_{0}^{\prime}=\left\{(x, y) \mid M(x, y) \in G_{0}\right\} .
\end{aligned}
$$

2. Let $\left(p_{1}, q_{1}\right) \in Z \times Z^{\prime}-G^{\prime}$, then the sets are defined:

$\mathrm{G}_{1}=\left\{\mathrm{M}(\mathrm{x}, \mathrm{y}) \mid \mathrm{M}(\mathrm{x}, \mathrm{y})=\mathrm{M}\left(\mathrm{p}_{1}, \mathrm{q}_{1}\right)\right\}$ and

$\mathrm{G}^{\prime}{ }_{1}=\left\{(\mathrm{x}, \mathrm{y}) \mid \mathrm{M}(\mathrm{x}, \mathrm{y}) \in \mathrm{G}_{1}\right\}$.

3. Let $\left(p_{2}, q_{2}\right) \in Z \times Z{ }^{\prime}-G^{\prime}{ }_{0} \cup G^{\prime}{ }_{1}$, then the sets are defined:

$\mathrm{G}_{2}=\left\{\mathrm{M}(\mathrm{x}, \mathrm{y}) \mid \mathrm{M}(\mathrm{x}, \mathrm{y})=\mathrm{M}\left(\mathrm{p}_{2}, \mathrm{q}_{2}\right)\right\}$ and

$\mathrm{G}_{2}^{\prime}=\left\{(\mathrm{x}, \mathrm{y}) \mid \mathrm{M}(\mathrm{x}, \mathrm{y}) \in \mathrm{G}_{2}\right\}$. 
4. The process continues until the first natural number $k$ such that $Z \times Z^{\prime}-G_{0}^{\prime} \cup$ $\mathrm{G}^{\prime}{ }_{1} \cup \mathrm{G}_{2}{ }_{2} \cup \ldots \cup \mathrm{G}^{\prime}{ }_{\mathrm{k}+1}=\varnothing$ is found. It can be demonstrated that sets $\mathrm{G}^{\prime}{ }_{\mathrm{i}}, \mathrm{i} \in$ $\{0, \ldots, \mathrm{k}\}$ generate a partition of set $\mathrm{Z} \times \mathrm{Z}$ '. We call the union $\cup_{\mathrm{i}} \mathrm{G}_{\mathrm{i}}{ }_{\mathrm{i}}$ a primitive

\section{partition.}

To relax the equality condition that is requested in the definition of sets $G_{i}, i \in$ $\{1, \ldots, k\}$, we set up the generalized sets $G_{i}{ }^{\varepsilon}$ as follows: $G_{i}{ }^{\varepsilon}=\{M(x, y) \mid \| M(x, y)-$ $\left.\mathrm{M}\left(\mathrm{p}_{\mathrm{i}}, \mathrm{q}_{\mathrm{i}}\right) \| \leq \varepsilon\right\}^{8}$, where $\|\bullet\|$ could be any metric. In this work we use the "Manhattan" metric, i.e. $\|(x, y, z)-,(p, q, r)\|=|x-p|+|y-q|+|z-r| ;|\bullet|$ denotes the absolute value.

It is more convenient to work with the co-domain of the Image Associated Function because it does not contain repeated elements thus allowing avoid excessive computing. For example, consider figure 2.

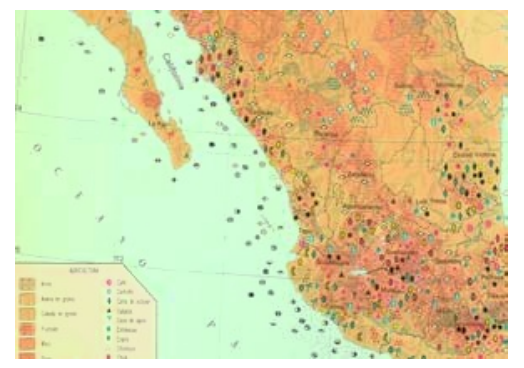

Fig. 2. Cartographic map: size $914 \times 539=492646$ pixels.

The number of elements in the co-domain of the Image Associated Function, considering the sets $\mathrm{G}_{\mathrm{i}}{ }^{\varepsilon}$, with $\varepsilon=20$, is 120 . In other words, we only use $0.02 \%$ of the total number of the image pixels to build primitive partition. To this partition, we apply the clustering criteria to extract and recognize the objects of interest in maps ${ }^{9}$.

Cartographic Pattern Recognition. Suppose that a cartographic pattern $\mathbf{P}_{\mathrm{TE}}$ already recognized by a computer system in a cartographic map in a coarse scale exists. The recognition of $\mathbf{P}_{\mathrm{TE}}$ implies that corresponding attributive, geometric, topologic data are known and, therefore, form the Cartographic Knowledge Domain (CKD). Information of special interest is its location with respect to some coordinate system, color or colors, shape, etc. We use this information to recognize or assign a concept from CKD, e.g. "Lerma River" to $\mathbf{P}_{\mathrm{FE}}$ presented in the fine scale map. Note that the characteristics of $\mathbf{P}_{\mathrm{TE}}$ and $\mathbf{P}_{\mathrm{FE}}$ are conceptually similar, e.g. they have the same name, although they can be distinct as functions according to definition 4 .

8 Notice that when $\varepsilon=0$, the sets $G_{i}^{\varepsilon}$ are converted in the sets $G_{i}$; that is why we call them "generalized".

9 Note that primitive partition in vector image of region maps, for example, is immediate and provides the region recognition as well, i.e. this is simultaneous segmentation-recognition [5] 
Information about the location of $\mathbf{P}_{\mathrm{TE}}$ is used to find a candidate to be "fine" cartographic pattern $\mathbf{P}_{\mathrm{FE}}$. Notice that $\mathbf{P}_{\mathrm{FE}}$ is generated by the application of the clustering criteria. This way, once $\mathbf{P}_{\mathrm{FE}}$ has been located the system is assigned to it the concept defined by "coarse" cartographic pattern $\mathbf{P}_{\mathrm{TE}}$, finally reaching $\mathbf{P}_{\mathrm{FE}}$ recognition under that concept. For example, suppose that a cartographic map in coarse scale provides information with which the following CKD is build: \{name: "Palm"; location: $(84,101)\}$. CKD affirms that it is possible to find in the image coordinates $(84,101)$ a cartographic pattern denominated "Palm". Notice that the location, extraction and recognition are immediate because the classification has been employed over all image pixels by means of the Image Associated Function co-domain; see figure 3.

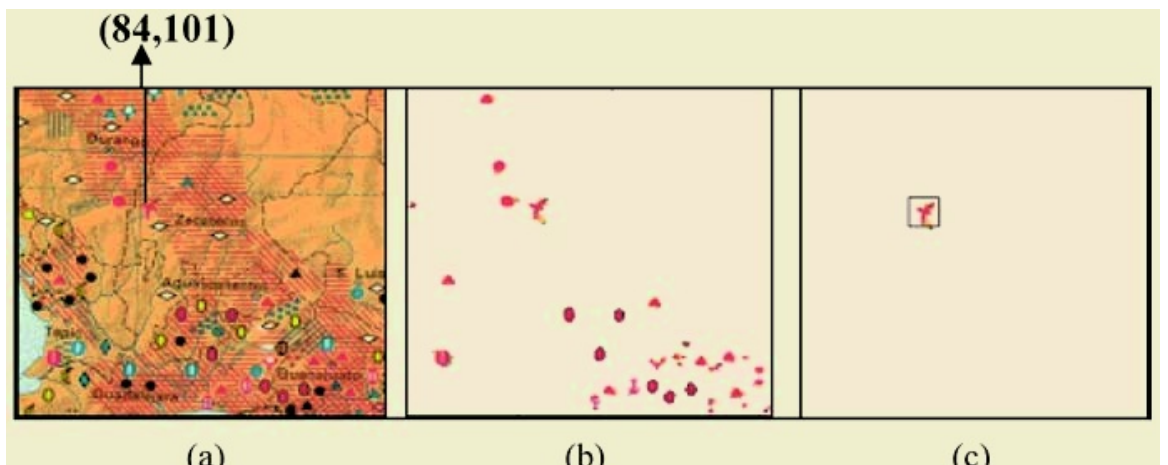

Fig. 3. Recognition of a cartographic pattern visually classified as punctual. (a) Original image. (b) Image obtained after applying the criterion $\beta_{\mathrm{o}}$-Connected, $\beta_{\mathrm{o}}=0.9$. (c) Recognition of the pattern (function) "Palm".

\section{Conclusion}

Color cartographic pattern recognition by a computer system remains a hard task [4]. This is closely related to the spatial data vectorization for GIS-ready information [2]. In this context, the coarse-to-fine scale method represents a promising alternative to carry it out. Indeed, a typical situation in GIS development is that spatial data in a coarse scale are available, and GIS-developer needs to transform (recognize) them into finer scale. For example, Mexico has full-territory coverage by recognized topographic maps in scale 1:50,000, but not in 1:25,000; this is a commonality for many countries [4]. Moreover, the C2FS method is in essence a simultaneous segmentation-recognition system [5][7].

The method attempts to incorporate the external (interpretative) knowledge to the segmentation-recognition process; an early approach with the same core was [10].

We believe that this work is not a yet another paper about (YAPA) cartographic pattern recognition. This assertion is also based on "universality" of our method: this was initially designed to recognize cartographic patterns into fine scale maps, how- 
ever, further development shown that it is possible to recognize patterns independently of the map scale by modifying the cartographic knowledge domain.

Of course, the method has certain limitations. One of them is that it is required a lazy user's intervention to establish the clustering criteria parameters. This common problem in image processing and pattern recognition, however, is not a great obstacle for further method's development by adding more pattern features to the criteria. Although, the method has actually been designed at basic level (considers unique color feature), requiring further development (consider other features such as shape), it is technically sound, according to the obtained results.

\section{References}

1. Martínez-Trinidad, J.F., Guzmán-Arenas, A.: The Logical Combinatorial Approach to Pattern Recognition, an Overview through Selected Works. Pattern Recognition, Vol. 34, No. 1. (2001) 741-751

2. Levachkine, S.: Raster to Vector Conversion of Color Cartographic Maps for Analytical GIS. In: Llados, J. (ed.): Proc. $5^{\text {th }}$ IAPR International Workshop on Graphics Recognition (GREC 2003) July 30-31, 2003, Barcelona, Catalonia, Spain (2003) 77-91

3. Martínez-Trinidad, J.F., Ruiz-Shulcloper, J.: Fuzzy Clustering of Semantic Spaces. Pattern Recognition, Vol. 34, No. 4. (2001) 783-793

4. Levachkine, S. P., Polchkov, E.A.: Integrated Technique for Automated Digitization of Raster Maps, Revista Digital Universitaria, Vol. 1, No. 1. (2000). Available on-line at http://www.revista.unam.mx/vol.1/art4/index.html

5. Levachkine, S., Torres, M., Moreno, M., Quintero, R.: Simultaneous SegmentationRecognition-Vectorization of Meaningful Geographical Objects in Geo-Images. Lecture Notes in Computer Science. Vol. 2905, Springer-Verlag (2003) 635-642

6. Angulo, J., Serra, J.: Mathematical Morphology in Color Spaces Applied to the Analysis of Cartographic Images. In: Levachkine, S., Serra, J., Egenhofer, M. (eds.), Semantic Processing of Spatial Data, Research on Computing Science, Vol.4. (2003) 59-66

7. Levachkine, S., Velázquez, A., Alexandrov, V., Kharinov, M.: Semantic Analysis and Recognition of Raster-scanned Color Cartographic Images. Lecture Notes in Computer Science, Vol. 2390. Springer-Verlag (2002) 178-189

8. Lazo-Cortés, M., Ruiz-Shulcloper, J., Alba-Cabrera, E.: An Overview of the Evolution of the Concept of Testor. Pattern Recognition, Vol. 34, No. 4. (2001) 753-762

9. Torres-Ruiz, M., Levachkine, S.: Semantics Definition to Represent Spatial Data. In: Levachkine, S. et al. (eds.), Proc. International Workshop on Semantic Processing of Spatial Data (GEOPRO 2002), 3-4 December 2002, Mexico City, Mexico (2002)

10. Meyers G.K., Chen, C.-H.: Verification-based Approach for Automated Text and Feature Extraction from Raster-scanned Maps. Lecture Notes in Computer Science, Vol. 1072. Springer-Verlag (1996) 190-203 